\title{
Generation of human-induced pluripotent stem cells from burn patient-derived skin fibroblasts using a non-integrative method
}

\author{
SHANGFENG FU ${ }^{1 *}$, JIANWU DING $^{2 *}$, DEWU LIU $^{1}$, HEPING HUANG $^{1,3}$, MIN LI $^{1,4}$, \\ YANG LIU ${ }^{1}$, LONGXIANG TU ${ }^{1}$ and DEMING LIU ${ }^{5}$ \\ ${ }^{1}$ Burns Institute, The First Affiliated Hospital of Nanchang University; ${ }^{2}$ Department of Oncology, \\ The Second Affiliated Hospital of Nanchang University; ${ }^{3}$ Department of Plastic and Aesthetic Surgery, \\ Jingxi Maternal and Child Health Hospital; ${ }^{4}$ Department of Obstetrics and Gynecology, The First Affiliated Hospital of \\ Nanchang University; ${ }^{5}$ Medical College of Nanchang University, Nanchang, Jiangxi 330006, P.R. China
}

Received March 17, 2017; Accepted October 19, 2017

DOI: $10.3892 /$ ijmm.2017.3206

\begin{abstract}
Patient specific induced pluripotent stem cells (iPSCs) have been recognized as a possible source of cells for skin tissue engineering. They have the potential to greatly benefit patients with large areas of burned skin or skin defects. However, the integration virus-based reprogramming method is associated with a high risk of genetic mutation and mouse embryonic fibroblast feeder-cells may be a pollutant. In the present study, human skin fibroblasts (HSFs) were successfully harvested from patients with burns and patient-specific iPSCs were generated using a non-integration method with a feeder-free approach. The octamer-binding transcription factor 4 (OCT4), sex-determining region Y box 2 (SOX2) and NANOG transcription factors were delivered using Sendai virus vectors. iPSCs exhibited representative human embryonic stem cell-like morphology and proliferation characteristics. They also expressed pluripotent markers, including OCT4, NANOG, SOX2, TRA181, stage-specific embryonic antigen 4 and TRA-160, and exhibited a normal karyotype. Teratoma and embryoid body formation revealed that iPSCs were able to differentiate into cells of all three germ layers in vitro and in vivo. The results of the present study demonstrate that HSFs derived from patients with burns, may be reprogrammed into stem cells with pluripotency, which provides a basis for cell-based skin tissue engineering in the future.
\end{abstract}

Correspondence to: Professor Dewu Liu, Burns Institute, The First Affiliated Hospital of Nanchang University, 17 Yongwai Street, Nanchang, Jiangxi 330006, P.R. China

E-mail: dewuliu@126.com

${ }^{*}$ Contributed equally

Key words: reprogramming, induced pluripotent stem cells, pluripotency, human skin fibroblast, burns

\section{Introduction}

Previous studies have suggested that somatic cells from mice and humans may be reprogrammed to become induced pluripotent stem cells (iPSCs) $(1,2)$. The reprogrammed cells were derived from a variety of different tissues and organs, including fibroblasts, keratinocytes and urine renal epithelial cells (3-7). The human skin fibroblast (HSF)-iPSCs demonstrated clear similarities with embryonic stem cells (ESCs) in their proliferation, pluripotency, clonal morphology, growth characteristics, surface markers, gene expression and epigenetics. HSF-iPSCs provide a reliable source of skin seed cells for specific patients, including individuals with large area burns or skin defects $(8,9)$. HSF-iPSCs do not have the same ethical or immune rejection issues that are associated with ESCs $(8,9)$. iPSCs may also be used in drug screening and experiments to aid the understanding of specific disease mechanisms (10-12).

In previous studies, somatic cells were transduced with vectors containing the human transcription factors NANOG, octamer-binding transcription factor 4 (OCT4), sex-determining region Y box 2 (SOX2), Kruppel-like factor 4 (Klf4) and c-Myc, which subsequently reprogrammed them to become iPSCs $(1,2)$. Initially, lentivirus and retrovirus were used as vectors to introduce key transcription factors into target cells and thus induce reprogramming into iPSCs $(13,14)$. However, lentivirus and retrovirus are integrating viruses (15), meaning that the virus gene may insert into the genome of the target cells, potentially causing the reactivation of transgenes, uncontrolled gene silencing and residual expression. These alterations may lead to undesirable consequences, which makes them unsuitable within a clinical setting (16). Using a non-integrating method is considered more appropriate and may eliminate the problem of insertional mutagenesis (17).

Previous research into epigenetics has confirmed that iPSCs retain the memory of epigenetic signatures from their original tissue and are more likely to differentiate toward donor-associated cells (18). Certain imprinted genes associated with growth, metabolism and neurological development of iPSCs and initial somatic cells share the same epigenetical and transcriptional statuses (19). The memory of the epigenetics 
may limit the full differentiation potential of iPSCs (18). In the present study, a non-integrating method was used to reprogram cells from the skin of patients with burns to generate iPSCs. The results of the present study may provide an experimental basis for the clinical use of iPSCs as seed cells.

\section{Materials and methods}

Isolation of fibroblasts and cell culture. Human dermal tissues were harvested from residual skin used for skin grafts in patients with burns. All protocols were approved by the Biomedical Ethics Committee of the Affiliated Hospital of Nanchang University (Nanchang, China) and written informed consent was obtained from all patients. Tissues were washed 2-3 times with penicillin/streptomycin and phosphate-buffered saline (PBS) (all from Beijing Solarbio Science and Technology Co., Ltd., Beijing, China) within a vertical clean bench. The tissues were subsequently cut into 2 x10 mm sections. The tissues were digested with $0.25 \%$ trypsinethylenediaminetetraacetic acid (EDTA) (Gibco; Thermo Fisher Scientific, Inc., Waltham, MA, USA) at $4^{\circ} \mathrm{C}$ for $\sim 5 \mathrm{~h}$. High glucose Dulbecco's modified Eagle's medium (DMEM) supplemented with $10 \%$ fetal bovine serum (FBS; both from HyClone; GE Healthcare Life Sciences, Logan, UT, USA) was added to terminate the digestion process. The epidermis and dermis were separated using tweezers and dermal tissues were sliced into sections $\left(0.5-1 \mathrm{~mm}^{3}\right)$. The distance between each section was $0.3-0.5 \mathrm{~cm}$, from which the fibroblasts came out. Culture dishes containing the dermal tissues were inverted for drying for $4 \mathrm{~h}$, following which high glucose DMEM with $10 \%$ FBS was added and cultured at $37^{\circ} \mathrm{C}$ for $24 \mathrm{~h}$ in an atmosphere containing $5 \% \mathrm{CO}_{2}$. The medium was replaced every 3 days. After 24 days cultured, the fibroblasts were isolated from the dermal sections by $0.25 \%$ trypsin-EDTA digestion.

Passage of fibroblasts. To prevent cell density from inhibiting growth and to obtain a larger number of proliferating cells, cells were subcultured when they reached $80-90 \%$ confluence. The medium was replaced and cells were detached with $0.5 \mathrm{ml}$ $0.25 \%$ trypsin-EDTA solution following washing with PBS. When the intercellular space increased and cells appeared round under an inverted phase contrast microscope (CTR6000; Leica Microsystems GmbH, Wetzlar, Germany), high glucose DMEM with $10 \%$ FBS was added to terminate digestion. The cells were centrifuged at $120 \mathrm{x} g$ for $5 \mathrm{~min}$ at room temperature. The supernatant was removed and the remaining cell deposit was resuspended in $9 \mathrm{ml}$ high glucose DMEM with $10 \%$ FBS and cultured at $37^{\circ} \mathrm{C}$ with $5 \% \mathrm{CO}_{2}$ following being subcultured at a rate of 1:3. The medium was replaced every 3 days.

Transduction of HSFs and generation of HSFs-iPSCs. At 2 days prior to transduction, HSFs at passage 3 were harvested and inoculated into 2 wells (1 well for counting and the other for transduction; $2.5 \times 10^{5}$ cells/well) of a 6 -well plate with high glucose DMEM and 10\% FBS. The cells were completely adhered and stretched on the day of transduction. When cells reached 50-80\% confluence thy were transduced using the CytoTune ${ }^{\mathrm{TM}}$-iPS 2.0 Sendai reprogramming vectors (ID Pharma Co., Ltd., Tsukuba, Japan) containing the human transcription factors NANOG, OCT4 and SOX2 (10 $\mu \mathrm{l}$ of each transcription tube). Cells were incubated at $37^{\circ} \mathrm{C}$ in an atmosphere containing $5 \% \mathrm{CO}_{2}$ overnight and the medium was replaced on the first day with fresh high glucose DMEM and $10 \%$ FBS to remove the Sendai reprogramming vectors. Following this, the medium was replaced every 2 days. At 6 days following transduction, the 6 -well plates were coated with 1\% Matrigel (BD Biosciences, Franklin Lakes, NJ, USA). At day 7 post-transduction, the cells were detached with $0.5 \mathrm{ml} 0.25 \%$ trypsin-EDTA solution following washing with PBS. When the cells were observed as round under the inverted phase contrast microscope, high glucose DMEM with $10 \%$ FBS was added to terminate the digestion. Cells were centrifuged at $200 \mathrm{x}$ g for $4 \mathrm{~min}$ at room temperature, following which the cell pellet was resuspended in $1 \mathrm{ml}$ high glucose DMEM with $10 \%$ FBS. The transduced cells were subsequently cultured in high glucose DMEM with $10 \%$ FBS under feeder-free conditions with $1 \%$ Matrigel-coated culture dish at a density of $1-5 \times 10^{5}$ cells $/ 100 \mathrm{~mm}$ at $37^{\circ} \mathrm{C}$ overnight in an atmosphere containing $5 \% \mathrm{CO}_{2}$. The medium was discarded the next day and replaced with reprogramming culture medium (ReproEasy; Beijing Cellapy Biotechnology Co., Ltd., Beijing, China). The medium was replaced every day and the culture was monitored until ESC-like colonies were observed under the inverted phase contrast microscope. At 4 weeks following transduction, the cell colonies were large and compacted enough to be picked out and expanded. They covered the majority of the surface area of the culture dish. The colonies were picked and transferred onto fresh $1 \%$ Matrigel-coated dishes with human PSCeasy medium (Beijing Cellapy Biotechnology Co., Ltd.) for expansion.

Picking out and transferring iPSCs colonies. Using a $1 \mathrm{ml}$ syringe, cell colonies were broken into pieces and transferred onto a $1 \%$ Matrigel-coated 6-well plate containing $1 \mathrm{ml}$ human PSCeasy medium. Plates were incubated at $37^{\circ} \mathrm{C}$ in a humidified atmosphere containing $5 \% \mathrm{CO}_{2}$. At $48 \mathrm{~h}$ post-transfer, colonies were attached to the culture plate and the medium was replaced. Following this, the medium was replaced every day. When the colonies covered $80-90 \%$ of the surface area of the culture plate they were considered ready for passaging.

Alkaline phosphatase (AP) staining. AP staining was performed using the BCIP/NBT Alkaline Phosphatase Color Development kit (Beyotime Institute of Biotechnology, Haimen, China) according to the manufacturer's protocol. Briefly, iPSCs were washed with PBS 3 times for 3 min each time. The iSPCs were fixed at room temperature in $4 \%$ paraformaldehyde (Beijing Dingguo Changsheng Biotechnology Co., Ltd., Beijing, China) for $20 \mathrm{~min}$ and washed again in PBS. BCIP/NBT solution was added to stain the iPSCs at room temperature for $20 \mathrm{~min}$ and the reaction was terminated by washing the cells twice with distilled water. Images were captured under an inverted phase contrast microscope (magnification, x100).

Immunofluorescence staining. A round coverslip was coated with $1 \%$ Matrigel overnight at $4^{\circ} \mathrm{C}$. The following day it was picked out and iPSC colonies were transferred onto it. The colonies were subsequently cultured with human PSCeasy medium at $37^{\circ} \mathrm{C}$ for $48 \mathrm{~h}$. When the colonies covered the 
Table I. Sequences of the primers used in the polymerase chain reactions.

Primer sequence $\left(5^{\prime}-3^{\prime}\right)$

\begin{tabular}{lllc}
\cline { 2 - 3 } Gene & \multicolumn{1}{c}{ Forward } & \multicolumn{1}{c}{ Reverse } & Size (bp) \\
\hline OCT4 & CCTCACTTCACTGCACTGTA & CAGGTTTTCTTTCCCTAGCT & 164 \\
GATA4 & GACAATCTGGTTAGGGGAAGC & GAGAGATGCAGTGTGCTCGT & 105 \\
MSX1 & TGCCTCGCTCTACGGTGCCT & GGCTGGAGGAATCGGCTGGC & 154 \\
SOX1 & TTTCCCTCGCTTTCTCA & TGCAGGCTGAATTCGGTT & 104 \\
GAPDH & GGAGCGAGATCCCTCCAAAAT & GGCTGTTGTCATACTTCTCATGG & 197 \\
\hline
\end{tabular}

OCT4, octamer-binding transcription factor 4; MSX1, Msh homeobox 1; SOX1, sex-determining region Y box 1; GAPDH, glyceraldehyde 3-phosphate dehydrogenase.

majority of the surface area, they were considered ready for immunofluorescence staining. The iPSC colonies were fixed with $4 \%$ paraformaldehyde at room temperature for $15 \mathrm{~min}$ and subsequently washed 3 times with PBS. Colonies were treated with $0.5 \%$ Triton $\mathrm{X}-100$ for $15 \mathrm{~min}$ at room temperature and blocked at room temperature using $3 \%$ bovine serum albumin (BSA; Sigma-Aldrich; Merck KGaA, Darmstadt, Germany) for $30 \mathrm{~min}$. Following removal of the blocking buffer, colonies were incubated with primary antibodies directed against, OCT4 (sc-9081; Santa Cruz Biotechnology, Inc., Dallas, TX, USA), TRA181 (MAB4381; EMD Millipore, Billerica, MA, USA), NANOG (ab109250; Abcam, Cambridge, UK), SSEA-4 (sc-21704; Santa Cruz Biotechnology, Inc.), SOX2 (630802; BioLegend, Inc., San Diego, CA, USA) and TRA-160 (sc-21705; Santa Cruz Biotechnology, Inc.) (all 1:100) at $4^{\circ} \mathrm{C}$ overnight with a blocking buffer (3\% BSA). Following washing with PBS three times, the colonies were incubated with secondary antibodies conjugated with Alexa Fluor 488 (A-11034) or Alexa Fluor 594 (A-11032) (1:100; both from Invitrogen; Thermo Fisher Scientific, Inc.) at $37^{\circ} \mathrm{C}$ for $50 \mathrm{~min}$ with a $3 \%$ BSA blocking buffer. The colonies were washed with PBS three times and a mounting medium with DAPI (Shanghai Yeasen Biotechnology Co., Ltd., Shanghai, China) was used to stain the cell nuclei. Images were captured within 30 min using the inverted phase contrast microscope.

Karyotyping. When iPSCs cultured in $60 \mathrm{~mm}$ dishes reached $80-90 \%$ confluence, they were treated with $50 \mathrm{ng} / \mathrm{ml}$ colcemid (Sinopharm Chemical Reagent Co., Ltd., Shanghai, China) for $7 \mathrm{~h}$ at $37^{\circ} \mathrm{C}$ before being harvested using trypsin-EDTA solution. The iPSCs were resuspended in $0.075 \mathrm{M} \mathrm{KCl}$ and incubated at $37^{\circ} \mathrm{C}$ for $20-40 \mathrm{~min}$, then fixed in 3:1 methanol: glacial acidic acid (both from Sinopharm Chemical Reagent Co., Ltd.) at room temperature for $10 \mathrm{~min}$. iPSCs were then centrifuged and fixed three times for ( $15 \mathrm{~min}$ at room temperature, $15 \mathrm{~min}$ at room temperature and overnight at $4^{\circ} \mathrm{C}$ ). The harvested cells were stained with Giemsa (Sinopharm Chemical Reagent Co., Ltd.) at room temperature for $5 \mathrm{~min}$. VideoTesT-Karyo 3.1 software (Leica Microsystems GmbH) was used to analyze the karyotype of iPSCs at passage 16.

Differentiation of iPSCs in vitro. When the iPSCs cultured in 6-well plates reached $80-90 \%$ confluence, they were harvested using EDTA solution (Beijing Cellapy Biotechnology Co., Ltd.) and resuspended with a differentiation medium [high glucose DMEM; 2 mM L-glutamine; $0.1 \mathrm{mM}$ nonessential amino acid (Invitrogen; Thermo Fisher Scientific, Inc.); $0.1 \mathrm{mM}$ b-mercaptoethanol (Sigma-Aldrich; Merck KGaA); 20\% FBS] at $37^{\circ} \mathrm{C}$ with $5 \% \mathrm{CO}_{2}$. The medium was refreshed every 3 days and the iPSCs were cultured in suspension. Following 7 days in suspension culture, embryoid bodies (EBs) had formed. The following day the EBs were transferred to $1 \%$ Matrigel-coated 6-well plates and cultured in the high glucose DMEM for 7 days at $37^{\circ} \mathrm{C}$ with $5 \% \mathrm{CO}_{2}$. The medium was refreshed every 2 days. The cells were harvested and specific gene expression was measured by polymerase chain reaction (PCR). This was done to demonstrate that the cells underwent spontaneous differentiation.

PCR. Following 14 days of incubation as described above, genomic DNA from iPSCs and EBs was extracted using a TIANamp Genomic DNA kit (Tiangen Biotech Co., Ltd., Beijing, China) according to the manufacturer's protocol. PCR was used to examine the expression of genes representative of the endoderm, mesoderm and ectoderm. The extracted genomic DNA of iPSCs and EBs was mixed with primers and a TIANamp Genomic DNA kit (Tiangen Biotech Co., Ltd.) and the thermocycling conditions were as follows: Pre-denaturation at $94^{\circ} \mathrm{C}$ for $4 \mathrm{~min}$, denaturation at $94^{\circ} \mathrm{C}$ for $30 \mathrm{sec}$, annealing at $55^{\circ} \mathrm{C}$ for $30 \mathrm{sec}$ and extension at $72^{\circ} \mathrm{C}$ for $30 \mathrm{sec}$ for 35 cycles, followed by a final elongation at $72^{\circ} \mathrm{C}$ for $2 \mathrm{~min}$ and storage at $4^{\circ} \mathrm{C}$. The amplified PCR products were resolved on $1.5 \%$ agarose gels (Thermo Fisher Scientific, Inc.) and glyceraldehyde 3-phosphate dehydrogenase (GAPDH) was used as an internal control. The gels were run for $25 \mathrm{~min}$ at $100 \mathrm{~V}$. Images were captured using a Bio-Rad Gel document system (Bio-Rad Laboratories, Inc., Hercules, CA, USA). The primer sequences of all primers are listed in Table I.

Teratoma formation. To evaluate the pluripotency of iPSCs in vivo, the HSF-iPSCs were harvested and suspended with PBS in a $1.5 \mathrm{ml}$ Eppendorf tube. A total of $1 \times 10^{7}$ cells were injected subcutaneously into the hind legs of 4-week-old male Non-obese diabetic-severe combine immune deficiency (SCID) mice ( $\mathrm{n}=7$; 28-35 days old; $15-17 \mathrm{~g}$; Charles River Systems, Inc., Burlington, MA, USA). Mice were housed at $22 \pm 2^{\circ} \mathrm{C}$ with $40-70 \%$ humidity and a $12 \mathrm{~h}$ light/dark cycle with free 

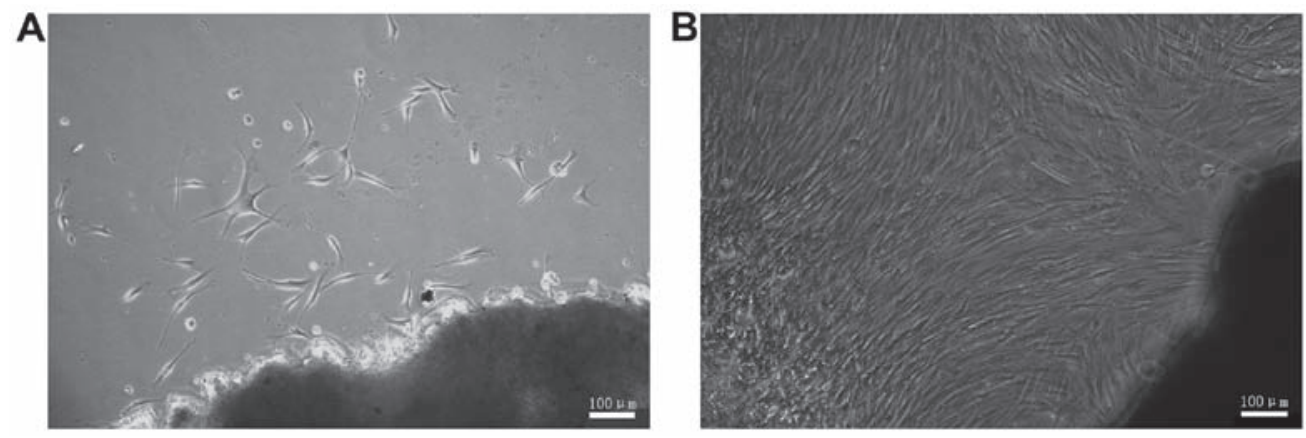

Figure 1. Morphology of human skin fibroblasts. Fibroblast cells following culture in high glucose Dulbecco's modified Eagle's medium with $10 \%$ fetal bovine serum for (A) 11 days and (B) 24 days.
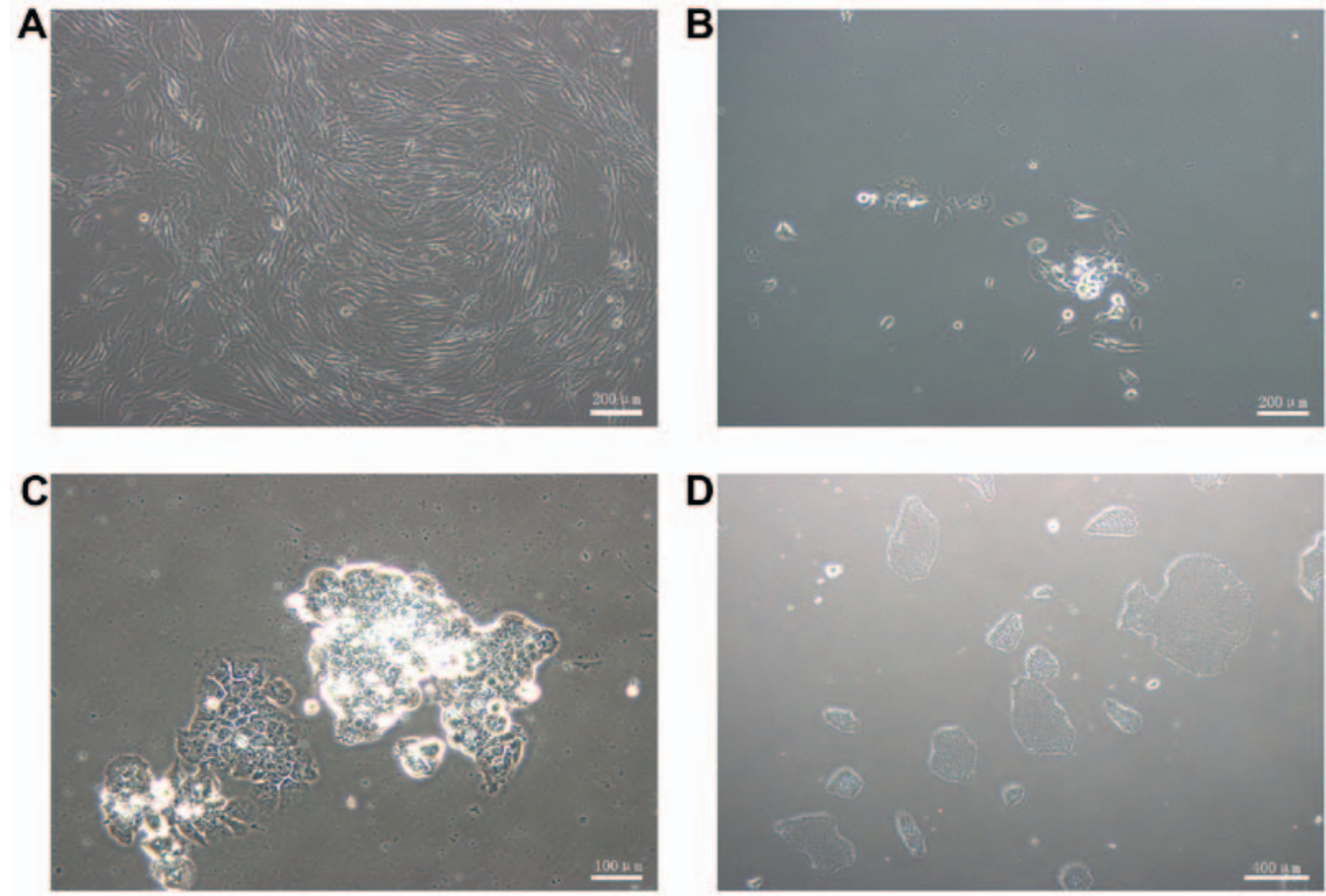

Figure 2. Generation of HSF-iPSCs. (A) Fibroblasts at passage 3 prior to transduction with Sendai virus and human transcription factors. (B) Embryonic stem cell-like colonies are visible 8 days following transduction. (C) HSF-iPSC colonies may be picked at week 4. (D) HSF-iPSCs at passage 2. HSF, human skin fibroblast; iPSCs, induced pluripotent stem cells.

access to food and water. At 8 weeks following injection, the formed tumors were dissected and harvested. The tumors were fixed in $4 \%$ paraformaldehyde at $4^{\circ} \mathrm{C}$ for 2 days, embedded in paraffin blocks, sliced into 3-4 $\mu \mathrm{m}$ sections and stained with hematoxylin and eosin (H\&E) staining for 5 min each at room temperature. Tissue samples were observed using a light microscope at x100 magnification. All animal experiments were approved by the Ethics Committee of the First Affiliated Hospital of Nanchang University.

\section{Results}

The morphological characteristics of HSFs. HSFs were isolated from the skin tissues of patients with burns and cultured in high glucose DMEM with $10 \%$ FBS. Following culturing for 11 days, it was possible to see fibroblast cells that had broken off from the tissue segment (Fig. 1A). The cells exhibited branch- and spindle-shaped morphology. Following culture for 24 days, the cells covered the majority of the surface area of the culture dish (Fig. 1B). Following passaging culture the HSFs demonstrated a marked proliferation ability.

Generation of HSF-iPSCs. To generate iPSCs using a non-integrating method, the HSFs were transduced with CytoTune-iPS 2.0 Sendai reprogramming vectors containing the human transcription factors NANOG, OCT4 and SOX2. The HSFs were cultured until they reached $50-80 \%$ confluence prior to transduction (Fig. 2A). At 7 days post-transduction, the cells were detached and transferred onto $1 \%$ Matrigel-coated $60 \mathrm{~mm}$ dishes. The following day, several small ESC-like colonies were observed (Fig. 2B). At 4 weeks post-transduction, the colonies exhibited representative human ESC-like morphology (Fig. 2C), which includes a large nucleoli and nucleus to cytoplasm ratio. The cells were packed tightly and the border was distinct. The ESC-like morphology and proliferation was maintained following 


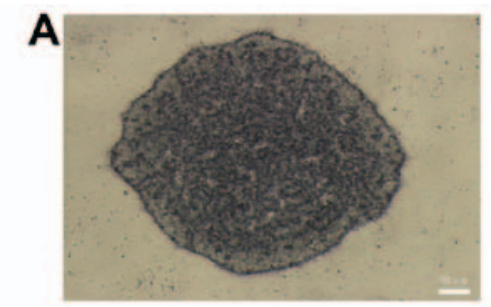

B

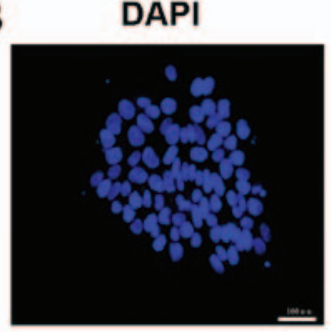

C

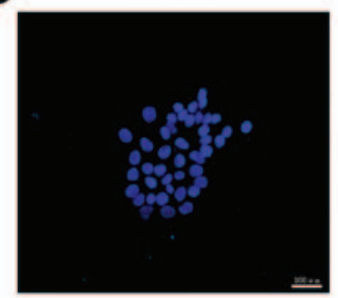

D

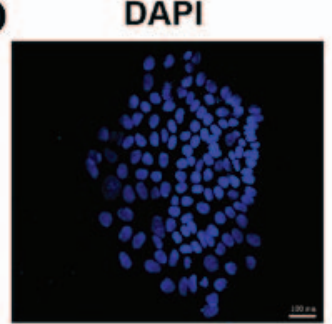

TRA-1-60

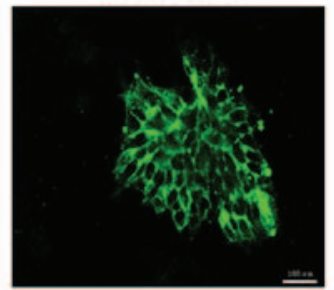

SSEA-4

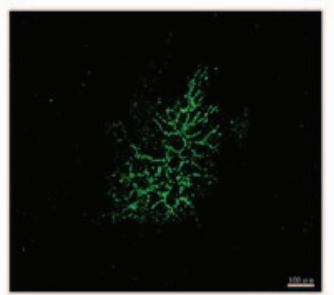

TRA-1-81

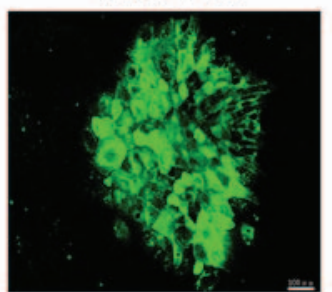

OCT4

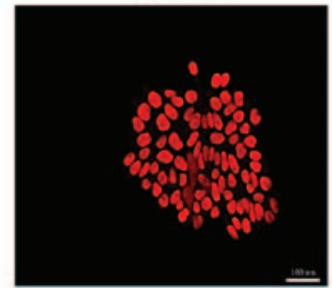

NANOG

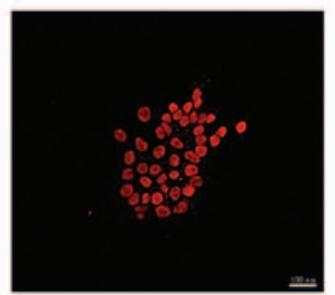

Sox2

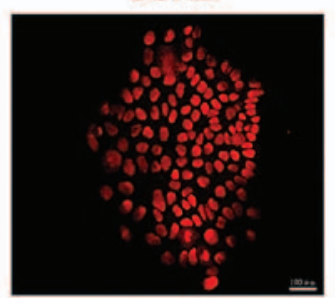

Merged

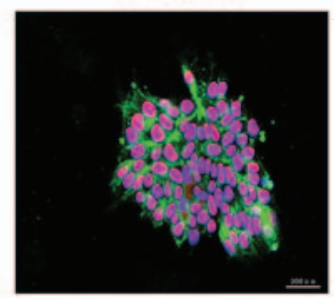

Merged

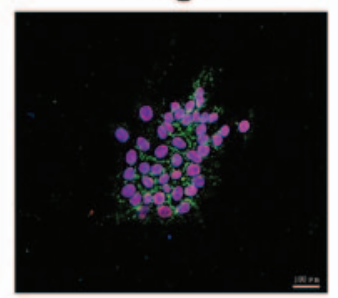

Merged

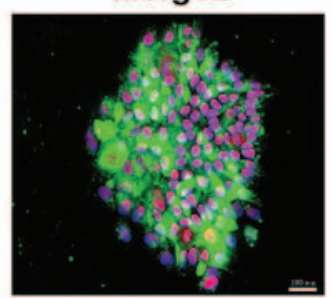

Figure 3. AP and immunofluorescence staining of HSF-iPSCs. (A) The HSF-iPSC colonies were positively stained with AP. Immunofluorescence staining was performed to detect pluripotent markers, including (B) TRA-1-60 and OCT4, (C) SSEA-4 and NANOG and (D) TRA-1-81 and SOX2. The nuclei of all cells were stained blue with DAPI. AP, alkaline phosphatase; HSF, human skin fibroblast; iPSCs, induced pluripotent stem cells; OCT4, octamer-binding transcription factor 4; SSEA, stage-specific embryonic antigen 4; SOX2, sex-determining region Y box 2.

passaging on $1 \%$ Matrigel-coated dishes with complete PSCeasy medium (Fig. 2D). A total of 100 ESC-like colonies per $1 \times 10^{5} \mathrm{HGFs}$ was obtained, therefore the reprogramming efficiency was $\sim 0.1 \%$.

Characteristics of HSF-iPSCs. The colonies exhibited positive AP staining (Fig. 3A), which indicated that pluripotent stem cells had been successfully developed. Immunofluorescence staining was used to examine the presence of pluripotency-associated proteins and revealed that the HSF-iPSCs strongly expressed surface pluripotency markers, including TRA181, SSEA-4 and TRA-160, as well as the intracellular pluripotency markers OCT4, NANOG and SOX2 (Fig. 3B-D). These results suggest that HSFs may be reprogrammed to form pluripotent stem cells. The HSF-iPSCs were confirmed by karyotype analysis (Fig. 4). They exhibited a normal karyotype of $46 \mathrm{XY}$ as confirmed by chromosomal G-band analysis at passage 16 .
Differentiation of HSFs-iPSCs in vitro and in vivo. To examine the differentiation potential of HSF-iPSCs in vitro, an experiment was designed to culture the HSF-iPSCs in suspension. The HSF-iPSCs differentiated to EBs spontaneously following 7 days in a suspension culture (Fig. 5A). The EBs were harvested and transferred to $1 \%$ Matrigel-coated 6-well plates and cultured for an additional 7 days in differentiation medium (Fig. 5B). DNA was isolated from the EBs and HSF-iPSCs and subsequently used for PCR to examine the expression of genes specific to the three germ layers. Msh homeobox 1 (MSX1; endoderm), GATA4 (mesoderm) and SOX1 (ectoderm) were revealed to be upregulated, whereas the ESC-specific gene OCT4 was downregulated in the EBs (Fig. 5C). Conversely, OCT4 was upregulated in HSF-iPSCs and MSX1, SOX1 and GATA4 were downregulated. The reference gene GAPDH was upregulated in the EBs and HSF-iPSCs. These results suggest that the HSF-iPSCs are capable of differentiating into various different cell types in vitro. 

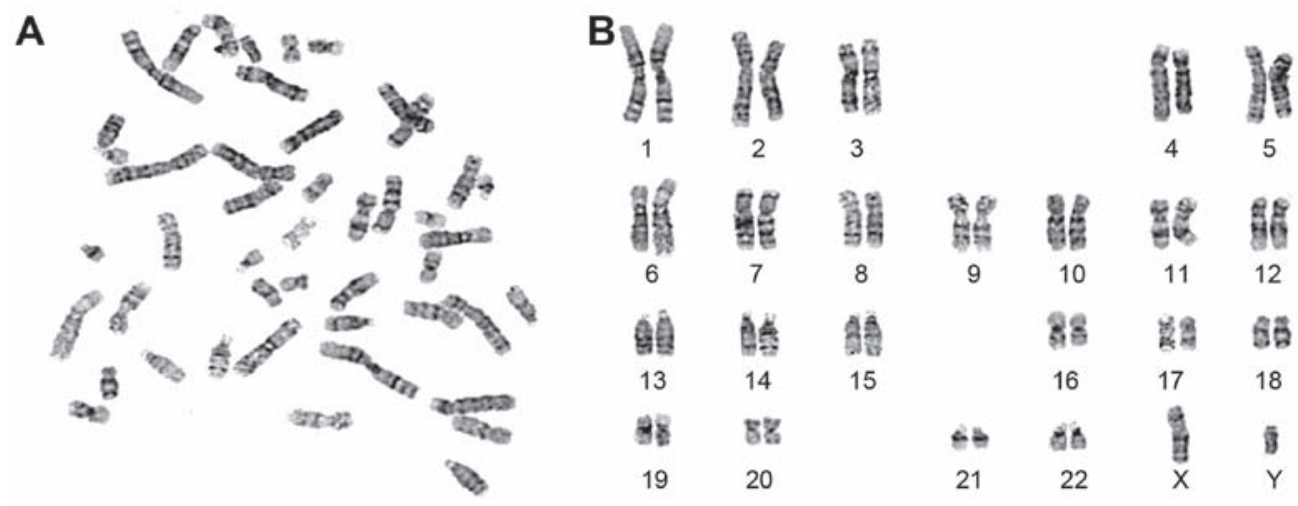

Figure 4. Karyotype analysis of human skin fibroblast-induced pluripotent stem cells. (A) Karyotype analysis revealed a normal karyotype of 46 chromosomes. (B) Analysis was organized according to the traditional format of chromosomes.
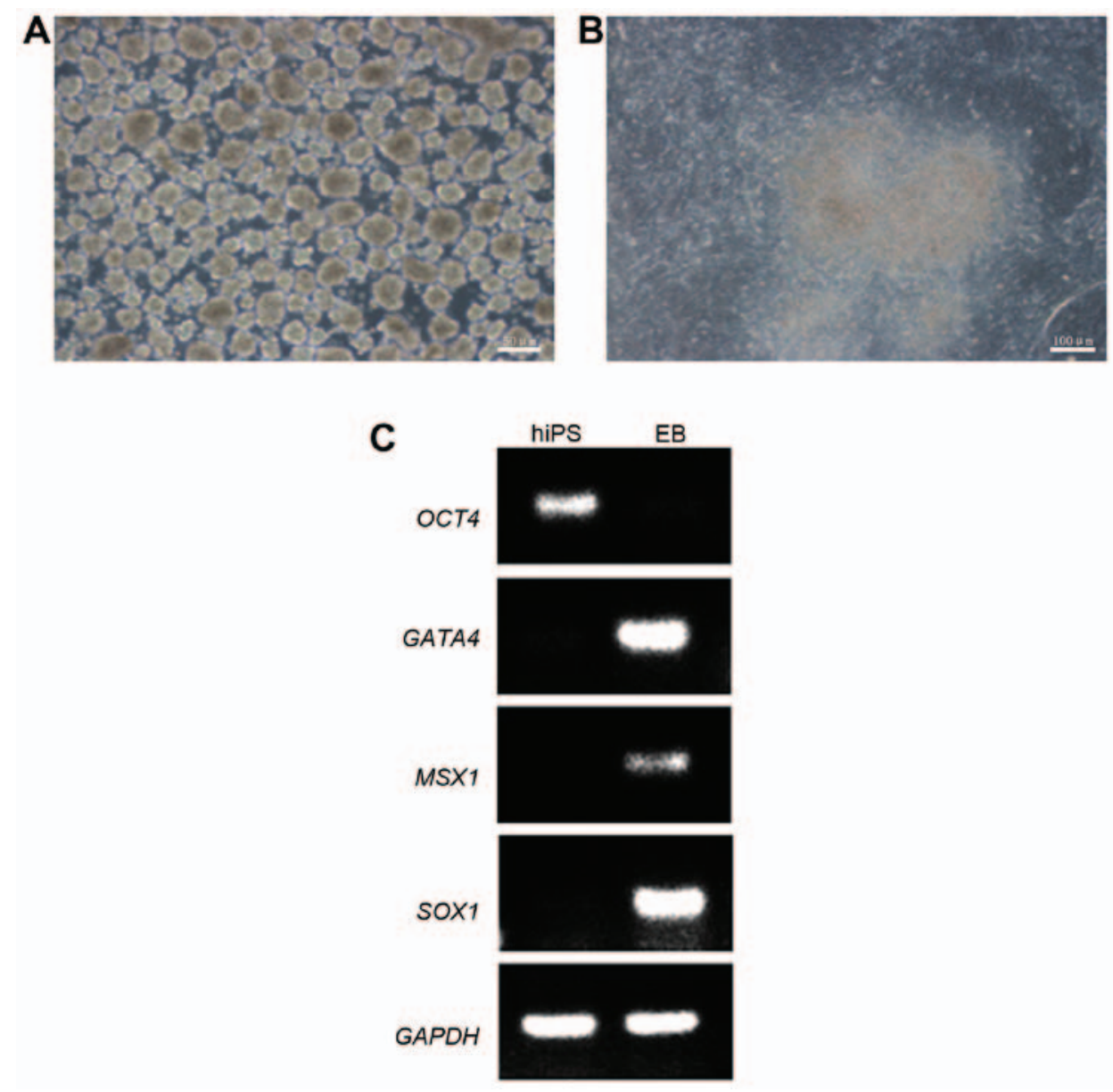

Figure 5. In vitro differentiation of HSF-iPSCs. (A) HSF-iPSCs were grown in suspension and EB formation was detected at 8 days. (B) EBs were grown in $1 \%$ Matrigel-coated 6-well plates for an additional 7 days and adherent cells were observed. (C) Polymerase chain reaction analysis was performed for differentiation markers of the three germ layers in HSF-iPSC clones and EBs. GAPDH was used as an internal control. HSF, human skin fibroblast; iPSCs, induced pluripotent stem cells; EB, embryoid bodies.

To examine the pluripotency of HSF-iPSCs in vivo, they were injected into the hind legs of SCID mice. At 8 weeks later, visible teratomas had formed (Fig. 6A and B). HE staining confirmed that the tumors contained derivatives of all three germ layers, including glands (endoderm), muscles (mesoderm) and nerves (ectoderm), as observed in Fig. 6C-E, respectively. These results indicate that HSF-iPSCs are able to differentiate into different cell types in vivo.

\section{Discussion}

Previous studies have successfully generated patient-specific iPSCs to treat a variety of diseases, including dystrophic epidermolysis bullosa, spinal muscular atrophy and Huntington's disease (20-22). In the present study, fibroblasts were isolated from the skin of patients with burns and patient specific iPSCs were developed following the reprogramming of fibroblasts. 

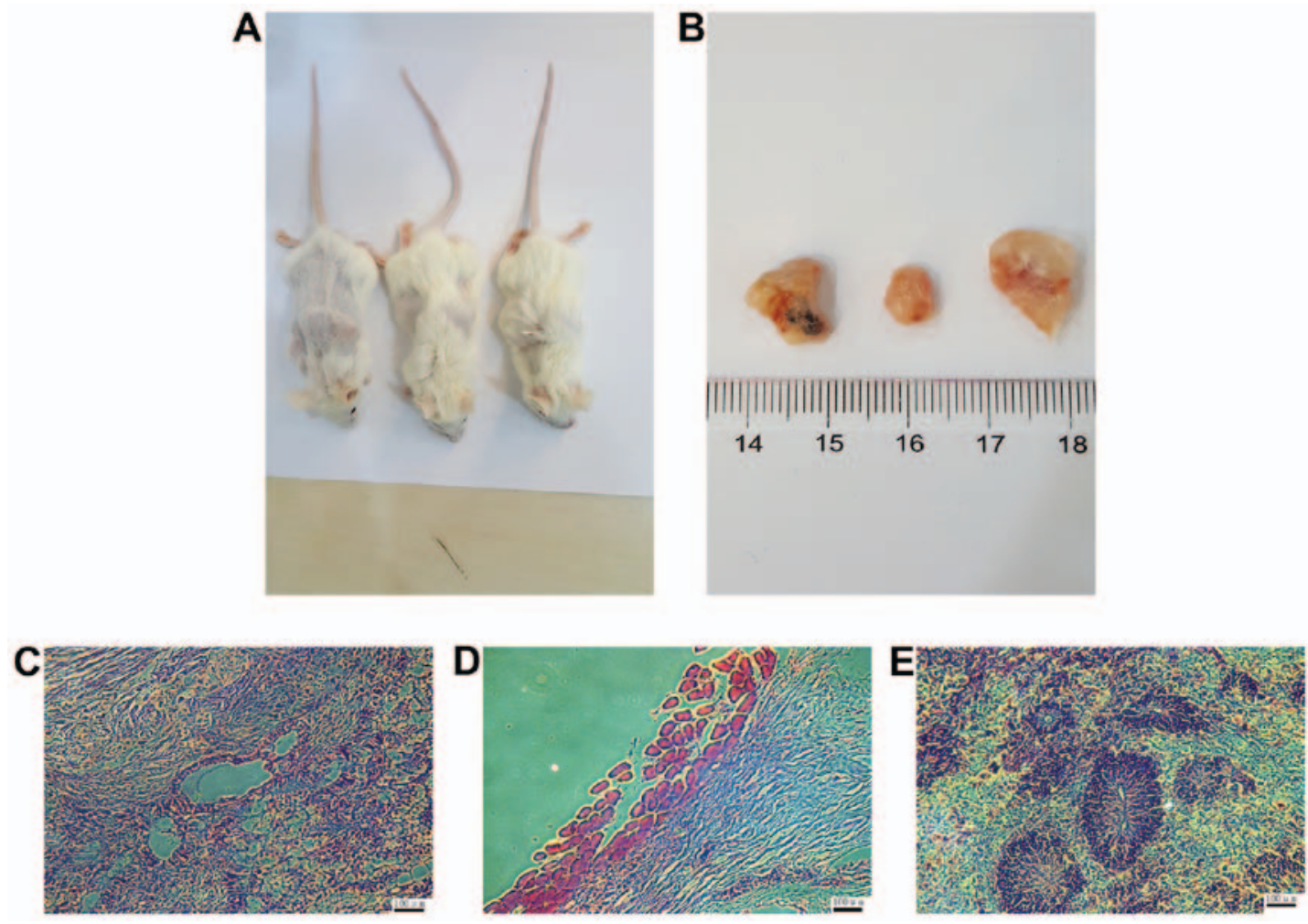

Figure 6. In vivo differentiation of HSF-iPSCs. (A) Injection of HSF-iPSCs into non-obese diabetic-severe combine immune deficiency mice gave rise to teratoma formation at 8 weeks. (B) The formed tumors were dissected from the mice. Hematoxylin and eosin staining of teratomas revealed the presence of differentiated tissues from all three germ layers, including (C) glands (endoderm), (D) muscles (mesoderm) and (E) nerves (ectoderm). HSF, human skin fibroblast; iPSCs, induced pluripotent stem cells.

Human dermal tissues were obtained from residual skin pieces following a skin graft on the patient with burns. Fibroblasts were harvested using the tissue block culture method and reprogrammed into iPSCs using the non-integration method. This process may provide a source of seed cells for patients with burns covering a large area, or individuals with skin defects.

Harvested cells demonstrated typical fibroblast morphology. In the present study, fibroblasts were transduced with Sendai virus reprogramming vectors containing the human transcription factors OCT4, SOX2 and NANOG, as opposed to OCT4, SOX2, Klf4 and c-Myc, as previous research has revealed that Klf4 and c-Myc are proto oncogenes, which may increase the tumor formation rate of iPSCs (23). The principal reason why the Sendai virus was selected to transduce the transcription factors was because it is a non-integrative virus and has a minimal effect on the cell genome following transduction (24-26).

Following transduction, the HSF-iPSCs morphology was observed as similar to ESCs. The immunofluorescence staining of the cells revealed the expression of pluripotency markers TRA181, SSEA-4, TRA-160, OCT4, NANOG and SOX2. Subsequently, it was demonstrated that cells were capable of differentiating into different cell types from the three germ layers in vitro and in vivo. These results suggest that HSF-iPSCs were successfully obtained. The HSF-iPSCs exhibited a normal karyotype of $46 \mathrm{XY}$ as demonstrated using chromosomal G-band analysis. To avoid the pollution of heterogeneous cells and improve the safety of iPSCs, they were cultured on $1 \%$ Matrigel-coated dishes instead of mouse embryonic fibroblast feeder-cells (27). In the present study, it was also revealed that skin tissue in skin grafts was thinner than regular skin tissue, as it did not contain subcutaneous tissue. The dermis and epidermis were isolated following 4-6 h digestion, which is a shorter time period than would be necessary for regular skin tissue and reduced the damage of the digestive enzymes to the cells (28).

The results of the present study demonstrate that fibroblasts harvested from patients with burns may be reprogrammed using Sendai virus vectors with OCT4, SOX2 and NANOG to form iPSCs with non-exogenous genomic integration $(17,29)$. HSF-iPSCs were demonstrated to be pluripotent and remain in an undifferentiated state. Further study is required to develop the differentiation of HSF-iPSCs into specific cells or tissues of the skin, including fibroblasts, keratinocytes, melanocytes or vascular tissue, lymphocytic tissue and nerves (30-34). The results of the present study provide an experimental basis for the development of functional skin within a laboratory for use in a clinical setting. The development of this novel treatment for disease or injury may be of great significance to regenerative medicine and tissue engineering.

\section{Acknowledgements}

The present study was supported by the National Natural Science Foundation of China (grant no. 81460293), the Science and Technology Planning Project of Jiangxi Province (grant no. 20133BBG70026) and the Special Fund for Graduate Innovation Project of Nanchang University (grant no. cx2016316). 


\section{References}

1. Takahashi $\mathrm{K}$ and Yamanaka S: Induction of pluripotent stem cells from mouse embryonic and adult fibroblast cultures by defined factors. Cell 126: 663-676, 2006.

2. Yu J, Vodyanik MA, Smuga-Otto K, Antosiewicz-Bourget J, Frane JL, Tian S, Nie J, Jonsdottir GA, Ruotti V, Stewart R, et al: Induced pluripotent stem cell lines derived from human somatic cells. Science 318: 1917-1920, 2007.

3. Huangfu D, Osafune K, Maehr R, Guo W, Eijkelenboom A, Chen S, Muhlestein W and Melton DA: Induction of pluripotent stem cells from primary human fibroblasts with only Oct4 and Sox2. Nat Biotechnol 26: 1269-1275, 2008.

4. Lowry WE, Richter L, Yachechko R, Pyle AD, Tchieu J, Sridharan R, Clark AT and Plath K: Generation of human induced pluripotent stem cells from dermal fibroblasts. Proc Nat Acad Sci USA 105: 2883-2888, 2008

5. Aasen T, Raya A, Barrero MJ, Garreta E, Consiglio A, Gonzalez F, Vassena R, Bilić J, Pekarik V, Tiscornia G, et al: Efficient and rapid generation of induced pluripotent stem cells from human keratinocytes. Nat Biotechnol 26: 1276-1284, 2008.

6. Sochacki J, Devalle S, Reis M, Mattos P and Rehen S: Generation of urine iPS cell lines from patients with attention deficit hyperactivity disorder (ADHD) using a non-integrative method. Stem Cell Res (Amst) 17: 102-106, 2016.

7. Xue Y, Cai X, Wang L, Liao B, Zhang H, Shan Y, Chen Q, Zhou T, Li X, Hou J, et al: Generating a non-integrating human induced pluripotent stem cell bank from urine-derived cells. PLoS One 8: e70573, 2013

8. Yamanaka S: Induced pluripotent stem cells: Past, present, and future. Cell Stem Cell 10: 678-684, 2012

9. Guha P, Morgan JW, Mostoslavsky G, Rodrigues NP and Boyd AS: Lack of immune response to differentiated cells derived from syngeneic induced pluripotent stem cells. Cell Stem Cell 12: 407-412, 2013.

10. Ishida Y, Kawakami H, Kitajima H, Nishiyama A, Sasai Y, Inoue $\mathrm{H}$ and Muguruma $\mathrm{K}$ : Vulnerability of purkinje cells generated from spinocerebellar ataxia type 6 patient-derived iPSCs. Cell Rep 17: 1482-1490, 2016.

11. Son MY, Kim YD, Seol B, Lee MO, Na HJ, Yoo B, Chang JS and Cho YS: Biomarker discovery by modeling Behçet's disease with patient-specific human induced pluripotent stem cells. Stem Cells Dev 26: 133-145, 2017.

12. Ye L, Chang JC, Lin C, Sun X, Yu J and Kan YW: Induced pluripotent stem cells offer new approach to therapy in thalassemia and sickle cell anemia and option in prenatal diagnosis in genetic diseases. Proc Natl Acad Sci USA 106: 9826-9830, 2009.

13. Takahashi K, Tanabe K, Ohnuki M, Narita M, Ichisaka T, Tomoda $\mathrm{K}$ and Yamanaka S: Induction of pluripotent stem cells from adult human fibroblasts by defined factors. Cell 131 861-872, 2007

14. Wilmut I: The first direct reprogramming of adult human fibroblasts. Cell Stem Cell 1: 593-594, 2007.

15. Okita K, Nakagawa M, Hyenjong H, Ichisaka T and Yamanaka S: Generation of mouse induced pluripotent stem cells without viral vectors. Science 322: 949-953, 2008.

16. Mack AA, Kroboth S, Rajesh D and Wang WB: Generation of induced pluripotent stem cells from $\mathrm{CD} 34^{+}$cells across blood drawn from multiple donors with non-integrating episomal vectors. PLoS One 6: e27956, 2011.

17. Fusaki N, Ban H, Nishiyama A, Saeki K and Hasegawa M: Efficient induction of transgene-free human pluripotent stem cells using a vector based on Sendai virus, an RNA virus that does not integrate into the host genome. Proc Jpn Acad Ser B Phys Biol Sci 85: 348-362, 2009

18. Kim K, Doi A, Wen B, Ng K, Zhao R, Cahan P, Kim J, Aryee MJ, Ji H, Ehrlich LI, et al: Epigenetic memory in induced pluripotent stem cells. Nature 467: 285-290, 2010.
19. Stadtfeld M, Apostolou E, Akutsu H, Fukuda A, Follett P, Natesan S, Kono T, Shioda T and Hochedlinger K: Aberrant silencing of imprinted genes on chromosome $12 \mathrm{qF} 1$ in mouse induced pluripotent stem cells. Nature 465: 175-181, 2010.

20. Itoh M, Kiuru M, Cairo MS and Christiano AM: Generation of keratinocytes from normal and recessive dystrophic epidermolysis bullosa-induced pluripotent stem cells. Proc Natl Acad Sci USA 108: 8797-8802, 2011.

21. Ebert AD, Yu J, Rose FF Jr, Mattis VB, Lorson CL, Thomson JA and Svendsen CN: Induced pluripotent stem cells from a spinal muscular atrophy patient. Nature 457: 277-280, 2009.

22. Tousley A and Kegel-Gleason KB: Induced pluripotent stem cells in Huntington's disease research: Progress and opportunity. J Huntingtons Dis 5: 99-131, 2016.

23. Borooah S, Phillips MJ, Bilican B, Wright AF, Wilmut I, Chandran S, Gamm D and Dhillon B: Using human induced pluripotent stem cells to treat retinal disease. Prog Retin Eye Res 37: 163-181, 2013.

24. Nishimura K, Sano M, Ohtaka M, Furuta B, Umemura Y, Nakajima Y, Ikehara Y, Kobayashi T, Segawa H, Takayasu S, et al: Development of defective and persistent Sendai virus vector: A unique gene delivery/expression system ideal for cell reprogramming. J Biol Chem 286: 4760-4771, 2011.

25. Lieu PT, Fontes A, Vemuri MC and Macarthur CC: Generation of induced pluripotent stem cells with CytoTune, a non-integrating Sendai virus. Methods Mol Biol 997: 45-56, 2013.

26. Churko JM, Burridge PW and Wu JC: Generation of human iPSCs from human peripheral blood mononuclear cells using non-integrative Sendai virus in chemically defined conditions. Methods Mol Biol 1036: 81-88, 2013.

27. Sun N, Panetta NJ, Gupta DM, Wilson KD, Lee A, Jia F, Hu S, Cherry AM, Robbins RC, Longaker MT, et al: Feeder-free derivation of induced pluripotent stem cells from adult human adipose stem cells. Proc Natl Acad Sci USA 106: 15720-15725, 2009.

28. Song Y, Ding J, Jin R, Jung J, Li S, Yang J, Wang A and Li Z: Expression and purification of FGF21 in Pichia pastoris and its effect on fibroblast-cell migration. Mol Med Rep 13: 3619-3626, 2016.

29. Zhao HX, Li Y, Jin HF, Xie L, Liu C, Jiang F, Luo YN, Yin GW, $\mathrm{Li}$ Y, Wang J, et al: Rapid and efficient reprogramming of human amnion-derived cells into pluripotency by three factors OCT4/SOX2/NANOG. Differentiation 80: 123-129, 2010.

30. Kogut I, Roop DR and Bilousova G: Differentiation of human induced pluripotent stem cells into a keratinocyte lineage. Methods Mol Biol 1195: 1-12, 2014.

31. Ohta S, Imaizumi Y, Okada Y, Akamatsu W, Kuwahara R, Ohyama M, Amagai M, Matsuzaki Y, Yamanaka S, Okano H, et al: Generation of human melanocytes from induced pluripotent stem cells. PLoS One 6: e16182, 2011.

32. Zanotelli MR, Ardalani H, Zhang J, Hou Z, Nguyen EH, Swanson S, Nguyen BK, Bolin J, Elwell A, Bischel LL, et al: Stable engineered vascular networks from human induced pluripotent stem cell-derived endothelial cells cultured in synthetic hydrogels. Acta Biomater 35: 32-41, 2016.

33. Khayyatan F, Nemati S, Kiani S, Hojjati Emami S and Baharvand $\mathrm{H}$ : Behaviour of human induced pluripotent stem cell-derived neural progenitors on collagen scaffolds varied in freezing temperature and laminin concentration. Cell J 16: 53-62, 2014.

34. Ando M, Nishimura T, Yamazaki S, Yamaguchi T, KawanaTachikawa A, Hayama T, Nakauchi Y, Ando J, Ota Y, Takahashi S, et al: A Safeguard system for induced pluripotent stem cell-derived rejuvenated T cell therapy. Stem Cell Reports 5: 597-608, 2015 .

This work is licensed under a Creative Commons Attribution-NonCommercial-NoDerivatives 4.0 International (CC BY-NC-ND 4.0) License. 